Itinéraires Itinéraires

Littérature, textes, cultures

\title{
Le sujet et son corps dans le roman marocain
}

\section{Khalid Zekri}

\section{OpenEdition}

\section{Journals}

Édition électronique

URL : http://journals.openedition.org/itineraires/1502

DOI : 10.4000/itineraires.1502

ISSN : 2427-920X

Éditeur

Pléiade

Édition imprimée

Date de publication : 1 novembre 2011

Pagination : 45-59

ISBN : 978-2-296-55720-8

ISSN : 2100-1340

Référence électronique

Khalid Zekri, « Le sujet et son corps dans le roman marocain », Itinéraires [En ligne], 2011-3 | 2011, mis en ligne le 01 novembre 2011, consulté le 30 avril 2019. URL : http://journals.openedition.org/ itineraires/1502 ; DOI : 10.4000/itineraires.1502

\section{c) () $\Theta$}

Itinéraires est mis à disposition selon les termes de la licence Creative Commons Attribution - Pas d'Utilisation Commerciale - Pas de Modification 4.0 International. 


\title{
Le sujet et son corps dans le roman marocain
}

\begin{abstract}
The body is part of the Moroccan literary texts through various figures: the feminine/ masculine, the madman, the homosexual, the prostitute, the believer, the illegal immigrant... In this article, we examine two records of the body: a first one is through the character homoerotic and the second one is through the female character. We analyze the writing of the body's feminine, the writing of homoeroticism, rites of passage and separation, the discourse on the margin as against speech, and, finally, the paradox of the writing of the censored body.
\end{abstract}

Keywords : body, Moroccan literature, gender, homoeroticism, censorship Mots clés : corps, littérature marocaine, genre, homoérotisme, censure

Le corps est assez souvent perçu au Maroc à partir d'une optique qui trace des lignes de démarcation entre le masculin et le féminin. Cette affirmation, fondée sur une acception dichotomique du genre, est aussi valable pour l'ensemble des pays du Maghreb avec plus ou moins de radicalité. Il s'ensuit que la construction sociale et anthropologique du corps devient déterminante dans la perception de soi; perception surdéterminée par le « corps prophétique » en tant que parangon qui structure l'imaginaire collectif du monde arabo-islamique en général. Wa lakum fi al-Rassûli iswatun hassana (le prophète est pour vous un bon exemple à suivre) est, du moins chez les sunnites, un syntagme qui participe amplement au sens que l'on donne à sa vie. Il est évident que ce syntagme fonctionne sur le mode d'un pouvoir discursif qui canalise le sens que les membres de la communauté musulmane donnent à leur vie. Les membres de cette communauté, sans respecter de la même manière ni avec la même rigueur les préceptes de l'islam, restent sous l'effet de l'exemplarité prophétique construite à partir, au moins, d'un double discours : exégétique (celui des théologiens et jurisconsultes) et culturel (celui des lettrés et de la vox populi). Sans que le corps de la femme ait un modèle codifié dans la culture arabo-islamique, les épouses et filles du prophète assument implicitement cette fonction. 
Le corps s'inscrit dans les textes littéraires marocains à travers plusieurs figures : le féminin/masculin, le fou, l'homosexuel, la prostituée, l'homme de religion, l'immigré clandestin... Nous privilégierons principalement deux types de personnages de récits littéraires à cause de leur dimension genrée : le personnage homoérotique et le personnage féminin.

\section{Écrire le corps au féminin}

La caractéristique principale du roman au féminin tel qu'il s'écrit au Maroc, est le passage obligé par le registre biographique ou autobiographique. La nouvelle et le roman constituent des genres littéraires vedettes parce qu'ils sont mieux appropriés à la mise en crise du vécu à travers leur dimension mimétique et les jeux de représentations que cette mimésis implique. Ces représentations permettent en effet de mettre en scène l'expérience du corps par rapport à la « horde tribale ». C'est, en effet, le corps de la femme qui écrit sur le vécu des femmes. Il y a un lien intime entre le corps écrivant et l'objet de l'écriture. Ce lien est déterminant aussi bien dans les caractéristiques énonciatives que dans l'élaboration de l'univers de la fiction. La consubstantialité entre sujet et objet de l'énonciation fait du texte romanesque un espace de commentaire. La femme, en se choisissant comme objet de narration, ne peut éviter des commentaires sur son rapport au monde. C'est ainsi que le roman au féminin se construit comme appropriation subjective du réel. La narratrice de Yawmiyat zawjat massoul $f i$ al-aryaf (Journal de l'épouse d'un responsable à la campagne) orchestre la distribution des voix narratives et fait passer son point de vue à travers des personnages auxquels elle donne la parole comme son mari ou le jardinier qui leur livre les secrets de la région dans laquelle cette narratrice va s'installer avec sa famille ${ }^{1}$. De plus, ce roman s'inscrit dans la mémoire de la littérature arabe moderne puisqu'il revendique, dès son titre, sa filiation avec Yawmiyat naib fi al-aryaf (Journal d'un substitut de campagne) de l'écrivain égyptien Tawfik Al-Hakim. La narratrice prolonge cette filiation de manière explicite dans le seuil du livre en se posant quelques questions dont la suivante : «Et pourquoi la narration ne serait-elle pas prise en charge par une épouse ${ }^{2}$ ? \Elle annonce son projet de mettre en scène le corps parlant d'un sujet féminin. Le monde échapperait ainsi à l'exclusivité du regard masculin pour être lu à travers le point de vue et la sensibilité de la femme.

Il en va de même pour la narratrice intradiégétique Leïla qui, dans Le Corps dérobé ${ }^{3}$, entreprend la douloureuse relation de sa vie. Elle concède très peu de perspectives aux autres personnages et apparaît ainsi dans l'économie générale du roman comme une narratrice souveraine qui filtre le

1. Dalila Hiyaoui, Yawmiyat zawjat massoul fi al-aryaf, Marrakech, Dar Al-Qoba azzarqa, 2000, p. 13.

2. Ibid., p. 5.

3. Houria Boussejra, Le Corps dérobé, Casablanca, Afrique Orient, 1999. 
monde à travers sa seule subjectivité. Mais ce droit à la subjectivité lui est refusé, non seulement par la société des hommes, mais surtout par les femmes : la mère, la fille et la belle-mère. Le corps de la femme devient ainsi objet de souffrances. C'est un corps coupable d'être au monde. Leïla a été méprisée car son corps a subi un viol dont elle n'est pourtant pas responsable. La narratrice devient ainsi clivée et le rapport qu'elle entretient avec son corps est marqué par une distance qui lui rend son appropriation problématique. Leïla refuse d'incorporer la passivité dans laquelle la société veut la maintenir. Elle fait sienne une corporalité rebelle qui trouve sa légitimité dans la lutte contre une société sans compassion pour la condition de la femme.

C'est ce qu'on trouve, sur un mode différent, chez Zhor Gourram. L'écriture du corps est affichée dès le titre de son roman Jassadun wa madinatun $^{4}$ (Corps et ville). La corporalité réfractaire de la narratrice trouve sa légitimité dans la décomposition de la ville imprégnée de valeurs négatives puisque « la ville et le corps se retrouvent dans la désagrégation » (p. 85). La corporéité n'est pas seulement une question anthropomorphique, mais aussi topographique puisque les corps narrés et les villes dans lesquelles ils se meuvent ne font plus qu'un seul Corps. Les villes revêtent parfois une dimension trans-territoriale car l'action du militant « idéaliste » Saïd, par exemple, est consubstantiellement liée aux résistants libanais et palestiniens. Le roman marocain écrit en langue arabe reste là encore lié au Machrek et à l'idéologie du panarabisme. Le rapport du corps à la ville n'est pas de connaissance mais de connivence. Le corps est ici le sujet de la perception. L'existence corporelle de la narratrice et des personnages évoqués dans le récit (Saïd, Ibrahim et Amal) réalisent l'unité d'un « entrer en soi » et d'un « sortir de soi » qui fondent la conscience de soi et sa projection dans les différentes villes constituant le support spatial de la narration. Le corps devient par là même une unité ouverte, une expressivité réciproque entre le monde et la conscience et, par conséquent, on peut conclure à une comparaison entre le corps et l'œuvre d'art suivant en cela Maurice Merleau-Ponty qui souligne que le corps « est un nœud de significations vivantes et non pas la loi d'un certain nombre de termes covariants ${ }^{5} \gg$. Ce fait n'est d'ailleurs pas récent puisque Platon assimilait déjà dans Phèdre corps et logos pour montrer que la rhétorique verbale divise le discours en tête, pieds, milieu et extrémités proportionnés. Cette métaphore renforce la fonction auctoriale qui sert de garant à l'intentionnalité littéraire du texte.

Soulignons par ailleurs que le roman au féminin est le récit d'une difficile intégration de la femme, comme sujet à part entière, dans la communauté. Les narratrices de Ni Fleur ni couronne, Rêves de femmes, L'Amande,

4. Zhor Gourram, Jassadun wa madinatun, Rabat, El Ghani, 1996.

5. Maurice Merleau-Ponty, Phénoménologie de la perception, Paris, Gallimard, 1945, p. 177. 
Jirah al-rrouh wa al-jassad (Blessures de l'âme et de la chair) ${ }^{6}$, Nadia dans Oser Vivre, Leilla dans Le Corps dérobé et Khadija dans Cérémonie racontent toutes, par l'entremise du flux de la conscience, l'abolition du lien entre elles et le monde ambiant. Même parmi des femmes, elles se sentent dans des orbites étrangères. Elles perçoivent les hommes, en règle générale, de manière dévalorisante. Il arrive qu'elles éprouvent de la répulsion à l'égard des hommes avec des degrés qui varient d'un récit à un autre car l'époux n'est pas toujours un soutien sûr. C'est souvent un obstacle à l'épanouissement corporel de la femme. C'est le cas de la narratrice de L'Amande par rapport à son premier mari et Nadia dans Oser Vivre par rapport à son époux qu'elle aime et qui l'a déçue. Dans l'économie générale du récit, le mari fonctionne souvent comme un moyen narratif qui participe, à son insu, au dévoilement du système phallocratique. La sur-admiration dont il bénéficie avant le mariage, s'effondre aussitôt après l'union institutionnelle. L'amour passe ainsi d'un traitement idéal à une mise à nu face aux épreuves de la réalité socioculturelle et aux pressions qui s'ensuivent.

Le roman au féminin montre que la culture livresque et la modernisation de la société ne sont pas suffisantes pour changer les mentalités. Les personnages masculins sont souvent des hommes cultivés (Ali dans Oser Vivre, Fadel dans Une femme tout simplement), mais la réalité dans laquelle ils vivent dépasse largement l'idéal de la modernité qui reconnaît à la femme le droit d'être un sujet à part entière. Aussi cultivé soit-il, le personnage masculin dans le roman marocain reste sous le joug de sa culture qui institue la domination masculine sur le corps de la femme. D'où ce mélange de complaintes du moi blessé et la mise en intrigue d'un désir de réalisation de soi qui finit par avorter. Là encore le commentaire intervient comme élément cardinal dans l'élaboration du roman. La vie des personnages est parsemée de commentaires qui marquent la fiction du sceau de l'engagement en faveur de la cause féminine, sans être pour autant une illustration du féminisme. Ces commentaires se situent parfois aux antipodes du féminisme moderniste comme c'est le cas dans Roju'ila al-tufula (Retour à l'enfance), Une enfance marocaine ou encore La Controverse des temps. Ce sont des romans qui introduisent le doute dans les promesses cathartiques de la modernité et constituent, avec des moyens narratifs de valeur inégale, une sorte de critique de la libération du sujet moderne par la raison.

Il s'ensuit que l'énoncé romanesque féminin se donne souvent dans un ton réfractaire et expose ainsi un éthos de la contestation à travers une rhétorique du corps rebelle. La mise en scène de cet éthos est liée à la nature même du matériau narratif surdéterminé par des biographèmes. Il s'agit donc d'une écriture qui puise sa matière narrative dans le vécu et revendique, par là même, son engagement. 


\section{Écrire l'homoérotisme}

Comme dans toutes les sociétés humaines dont la codification de l'hexis corporelle est saturée, il y a toujours des rituels sociaux et anthropologiques qui canalisent les comportements considérés comme déviants par rapport à cette codification. C'est le cas au Maroc du Moussem de Sidi Ali (festival annuel à caractère hagiographique), non loin de Moulay Idriss Zerhoun, qui permet la transgression de l'héteronormativité à partir (entre autres) de la célébration réelle ou simulée des unions homosexuelles. L'ordre masculin condensé dans le vocable homme, consubstantiellement lié à la virilité, se trouve par là même remis en question. Cette remise en question est également thématisée dans la littérature à travers quelques récits dont le sujet principal est la vie homosexuelle du narrateur et des personnages. Cette mise en relief de la masculinité comme construction sociale est exprimée dans L'Armée du salut d'Abdellah Taïa par le narrateur autofictionnel Abdellah quand il évoque le mariage de son frère Abdelkébir. Mariage qui est à l'origine de leur séparation affective puisqu'il est vécu par Abdellah comme un départ, une trahison imposée par les codes sociaux :

Un jour il est parti. Il s'est marié [...]. C'était une trahison, non de sa part, mais de la part de la société : un homme, un vrai, doit se marier ${ }^{7}$.

Chez Rachid O., la masculinité devient un lieu énonciatif d'interrogation à partir de la posture sexuelle. Dans L'Enfant ébloui, le narrateur met en relief la masculinisation de l'homosexualité active et la féminisation de l'homosexualité passive à travers sa relation amoureuse avec Khalil :

Il me disait : « tu es doux, tu es doux, comme une fille » ou « comme une petite fille ». La chose que j'ai pensée, sur le moment, c'est que je ne savais pas s'il le disait parce qu'il le pensait ou parce qu'il prenait plaisir à être avec moi et que, si j'étais doux comme une fille, alors ce n'était pas un problème de faire l'amour avec moi [...]. Pour moi, on faisait l'amour. Pour lui, il me faisait l'amour ${ }^{8}$.

Ou encore dans Chocolat chaud dédié à « Mathieu » :

[Mes camarades] trouvaient tous que j'étais d'une propreté étonnante et trop élégant pour un garçon, et tout l'effort que ma Lalla faisait pour mon apparence était pour eux comme un signe qui me rendait efféminé ${ }^{9}$.

7. Abdellah Taïa, L'Armée du salut, Paris, Seuil, 2006, p. 37.

8. Rachid O., L'Enfant ébloui, Paris, Gallimard, coll. «L'Infini », 1995, p. 45-46. Nous pouvons supposer qu'il s'agit probablement de Mathieu Lindon qui était, comme le dit René de Ceccatty dans sa préface au livre de A. Taïa, Mon Maroc, à l'initiative de la publication de Rachid O. Soulignons par ailleurs que dans Chocolat chaud, la figure paternelle est évoquée positivement, contrairement à Karim Nasseri pour qui le conflit avec le père constitue un moteur d'écriture.

9. Rachid O., Chocolat chaud, Paris, Gallimard, coll. « L'Infini », 1998, p. 57-59. 
La société est donc dans une logique de classification naturelle de l'homme et de la femme, du féminin et du masculin, de l'homo et de l'hétéro. Le seul usage d'un marqueur masculin (garçon ou homme) rappelle à tout individu de sexe masculin son statut viril. Nul besoin de justifier son statut puisque l'interpellation par le mot garçon (par rapport à fille et non par rapport au langage communément utilisé dans le milieu gay) ou homme renvoie aux codes sociaux qui distribuent les rôles et les places selon le genre. La femme finit par intérioriser cette domination masculine au point d'attendre de l'homme de faire d'elle une femme :

Non, je n'ai pas aimé Hmed, mais j'ai cru qu'il allait au moins me servir à quelque chose : faire de moi une femme. M'affranchir et me couvrir d'or et de baisers. Il n'a réussi qu'à me dépouiller de mes rires ${ }^{10}$.

Le discours de la narratrice, ne fait que reproduire la loi du monde social qui « construit le corps comme réalité sexuée et comme dépositaire de principes de vision et de division sexuants ${ }^{11} »$.

\section{Rites de passage et de séparation}

Le refus que la communauté oppose aux pratiques qui ne sont pas hétéronormatives n'est pas fondé sur le cumul et la formation d'un savoir susceptible de l'instruire sur ses propres marges. Les marges de la société marocaine, à commencer par le corps, relèvent de son impensé culturel. Le corps de l'enfant, tel qu'il est représenté par les auteurs cités, est soumis à une technologie du pouvoir social à travers la circoncision et l'expulsion du hammam des femmes dès qu'il commence à se faire remarquer par cellesci, et notamment par la tenancière du hammam. Le marquage de la verge par l'acte de circoncision trouve ses racines dans l'étymologie du lexique arabe puisque le mot 'odhra (prépuce) signifie empreinte ou indice et rejoint par là même le sens de la Khutûna en tant que marquage. Il serait même intéressant, comme le souligne Malek Chebel, d'établir un lien étymologique entre le verbe 'adhara qui a " aussi le même sens : "stigmatiser", "marquer" [...] et le fait de se marier (khutûna) car ils dérivent de la même racine kh-t- $n^{12}$ ».

Le vocabulaire est donc lui-même porteur d'un pouvoir coercitif sur le corps arabo-musulman puisqu'il lui imprime le tatouage de l'hétéronormativité dès le jeune âge. Le corps est ainsi « normé » et « sociabilisé » à travers des rituels dont le choix échappe au sujet qui les subit. Le corps est ainsi assujetti à la loi de la communauté qui tente de le priver de son autonomie.

10. Nedjma (pseudonyme), L'Amande, Paris, Plon, 2004, p. 47. En italique dans le texte.

11. Pierre Bourdieu, La Domination masculine, Paris, Seuil, coll. «Liber », 1998, p. 16.

12. Malek Chebel, Histoire de la circoncision, Paris, Balland, 1997, p. 55. Voir aussi un autre livre du même auteur : L'Esprit de sérail [1988], Paris, Payot/Rivages, 1998. Dans Histoire de la circoncision (p. 51-64), Malek Chebel a nuancé certaines de ses analyses concernant la circoncision qu'il a développées dans L'Esprit de sérail (p. 207-228). 
Cet assujettissement ne garantit cependant pas de manière absolue l'efficacité de ce rite : la circoncision tente d'assigner au jeune garçon un sexe masculin, mais elle n'a pas toujours le pouvoir de sexuer sa vie psychique. Les personnages sont mis en scène en tant que sujets dont 1'orientation sexuelle est marquée du sceau de la masculinité qui est nettement distinguée de la féminité, historiquement dominée par les lois du système phallocratique. Cette identité sexuelle imposée socialement dénie toute possibilité d'opter pour d'autres pratiques ou d'autres appartenances sexuelles que celles préalablement codifiées.

Ce marqueur d'appartenance sexuelle tente d'éviter toute ambiguïté possible car le garçon est censé agir socialement en fonction de son sexe que la loi de la communauté confond avec son genre. L'acte de circoncision joue le rôle d'un acte de langage performatif puisqu'il permet d'effectuer ce qu'il nomme : circoncire un garçon c'est, entre autres, l'inscrire dans une chaîne d'interpellations par lesquelles sa masculinité est rituellement fixée. Ce marquage du corps du garçon par l'hétéronormativité, censée orienter son désir, est également appuyé par le Coran qui distribue de manière claire (et dans une logique relevant de la nature) les rôles sexuels. Il fixe par là même un ordre sexuel que le sujet doit intérioriser pour se positionner dans sa société : «Ainsi, vous allez vers les hommes avec désir au lieu d'aller vers les femmes, mais ne savez-vous pas que vous êtes dans l'égarement ${ }^{13}$ ? » Le Coran, en tant que discours social porteur d'un message religieux, et donc d'une prescription morale, censure tout ce qui n'est pas hétéronormatif. Cela ne va pas sans produire un trouble psychique, aussi relatif soit-il, chez le sujet comme en témoigne L'Enfant ébloui de Rachid O. Dans ce récit, le lien que le sujet de l'énonciation établit entre sa subjectivité et Dieu apparaît dans le troisième chapitre intitulé, à juste titre, « Musulman » :

\footnotetext{
À un moment, j'ai lu des livres sur l'enfer et le paradis, et ce qui pouvait se passer en enfer me terrorisait, ça me rendait mal. Maintenant, je ne me pose même plus la question, car, si je me la posais vraiment, je ne pourrais plus vivre ma vie du tout, ma vie privée, ma sexualité me seraient complètement interdites ${ }^{14}$.
}

En faisant une entorse à l'ordre religieux et social, l'auteur déstabilise en même temps l'ordre politique qui assigne à chaque sexe son identité et à chaque individu le comportement correspondant à sa place dans la hiérarchie sociale. L'ordre politique, qui fonctionne toujours selon la logique de la normativité, se trouve donc remis en question et confronté à un désordre sexuel : il s'agit avant tout de subvertir la politique de la norme. Mais le discours coranique n'est pas le seul formateur de la sexuation du sujet. Le rite du hammam permet également à l'enfant d'avoir un statut qui lui reconnaît son appartenance sexuelle à l'univers masculin. La

13. Coran, selon la traduction de Kasimirski, Sourate XXVII, Verset 55.

14. Rachid O., L'Enfant ébloui, op. cit., p. 126. 
topique du hammam constitue à la fois un rite de passage et de séparation puisque le jeune narrateur raconte la douleur occasionnée par ce changement. En effet, chasser l'enfant du hammam des femmes, ce n'est pas seulement couper le cordon « sensitif » qui le lie à sa mère (ou à son substitut), mais surtout le priver de toute possibilité de mise en discours et d'identification au sexe féminin. Interdire à l'enfant de voir la nudité féminine, c'est aussi chercher à bien garder le secret du corps féminin pour mieux le protéger du désir masculin. Dans ce cas, le passage du hammam des femmes à celui des hommes occasionne un choc chez le jeune garçon, sans constituer forcément une inhibition :

À six ans, je n'avais plus le droit de rentrer [au hammam des femmes], j'ai commencé à aller avec mon père. Au début, on m'a dit que je pleurais parce que, la première fois, je n'avais pas envie d'aller avec mon père. À l'entrée du hammam, il y a deux portes collées, Hommes et Femmes. C'est un choc pour un enfant de ne plus entrer par la porte à côté où il a passé longtemps ${ }^{15}$.

L'interdiction d'accès au hammam des femmes signifie au jeune garçon qu'il est devenu homme, avec toute la connotation culturelle qu'implique ce mot (virilité, vigueur et puissance sexuelles...) : le but est de le renforcer dans la conscience de son sexe masculin. Mais, 1'enfant peut parfois prolonger son lien avec « la nudité » féminine que lui propose le hammam :

Quand j'étais enfant, j'étais insolent et perturbateur. J'allais tous les jours au hammam des femmes et m'accroupissais à genoux, sous l'ombre à côté de la porte. J'observais des femmes jeunes et vieilles se promener nues ou presque nues avec leurs seaux d'eau ${ }^{16}$.

L'univers des femmes lui est ainsi interdit d'accès car la communauté des femmes a décidé que son sexe était devenu incontrôlable. On lui reconnaît par là même une masculinité liée à l'intérêt qu'il commence à porter (en tant qu'enfant) à son sexe. Ainsi, à travers la gestion féminine du hammam, le pouvoir fait jouer sa « loi de prohibition ${ }^{17}$ » sur le sexe. Le corps du jeune garçon devient assujetti, c'est-à-dire soumis à la loi de la distinction des sexes et produit comme sujet masculin. La sexualité du sujet reste marquée par cet espace vaporeux car « tout un pan de la

15. Ibid., p. 31 .

16. Ibrahim Bouzalim, Ramadu Souss (Cendres du Souss), Marrakech, Dar Oualili, 1999, p. 39. Rachid O. développe la même thématique dans L'Enfant ébloui, p. 32 : « Dans le hammam des hommes, il y a un mur qui sépare du hammam des femmes. Juste en haut du mur, il y a un trou d'aération, bien haut pour que les hommes ne puissent pas y grimper. Et moi je me mettais contre le mur, tout contre, souvent j'étais fatigué à cause de la chaleur, souvent je m'allongeais par terre contre ce mur pour écouter parce que ça me manquait. Souvent, je m'endormais. »

17. Michel Foucault, Histoire de la sexualité, t. 1, La volonté de savoir, Paris, Gallimard, coll. « Bibliothèque des Histoires », 1976, p. 110. 
vie sexuelle s'organise en effet autour du hammam, le réel et le refus du réel, l'enfance et la puberté, le passage et l'initiation s'intègrent dans une espèce de constellation de sens que cristallise le hammam ${ }^{18} »$.

L'expulsion du hammam des femmes et le passage à celui des hommes accorde au narrateur une identité sexuelle non négociable puisqu'elle est fixée une fois pour toutes par des codes sociaux précis que l'école coranique, en tant que lieu de savoir fondé sur le Livre Absolu, ne cesse de renforcer. Tout cela fonctionne comme une norme coercitive porteuse, comme le dit à juste titre Michel Foucault dans un tout autre contexte, d'une " prétention de pouvoir ${ }^{19}$ ». Ce pouvoir, qui détient par ailleurs en lui-même ses principes de transformation et d'innovation, est fondé sur des mécanismes qui fabriquent et produisent un sujet hétéronormatif. Les récits évoqués ci-dessus subvertissent ces deux rites (circoncision et hammam) qui rappellent à tout porteur de sexe masculin son hétéronormativité.

Il n'en demeure pas moins que les modèles hétéronormatifs auxquels se dérobe le sujet de l'énonciation, exercent une violence symbolique sur lui puisqu'ils lui assignent, dès le plus jeune âge, un rôle sexuel préétabli par un consensus communautaire. D'où, à titre d'exemple, la visite que fait le jeune Idriss à un bordel féminin situé à Oujda. C'est son oncle qui l'y a conduit pour découvrir le sexe des femmes. Cette initiation a pour but de l'inscrire publiquement dans le registre de la virilité en mettant en valeur son hétérosexualité car, comme le lui dit son oncle :

pour bien mériter sa place au paradis de Dieu le Clément, l'homme se doit d'honorer sa femme. Nous n'avons pas encore l'âge de mariage mais les femmes que nous allons voir aiment bien qu'on soit à la hauteur. Tu es jeune et plein de fougue, alors je compte sur toi ${ }^{20}$.

Mais en même temps le jeune Idriss remet en question l'ordre hétéronormatif. Dans Noces et funérailles, il prend ce risque à travers un discours intérieur qui est marqué par l'usage de l'italique dans le texte :

Un homme? Qu'est-ce qu'un homme? Celui qui monte une femelle pour avoir une marmaille? Non, ma mère, je n'aime pas coucher avec une femme. L'imam de l'école coranique m'a initié à autre chose ${ }^{21}$.

Cette interrogation sur l'essence même de l'homme, entendu comme sexe masculin, remet en question la naturalisation des pratiques hétéronormatives. Mais, introduire un désordre dans le commun de la communauté n'expose-t-il pas le sujet à un risque de mise à l'écart et ne le relègue-t-il

18. Abdelwahab Bouhdiba, La Sexualité en Islam, Paris, PUF, coll. « Quadrige », 1975, p. 207.

19. Michel Foucault, Les Anormaux, Paris, Seuil/Gallimard, 1999, p. 46.

20. Karim Nasseri, Noces et funérailles, Paris, Denoël, 2001, p. 81.

21. Ibid., p. 14. 
pas, par là même, au rang des anormaux, et donc à la marge de la société? Il s'agit pour Idriss de mettre en crise cette naturalisation qui nie la circulation des désirs au profit de comportements et de pratiques communément admis comme naturels et normaux. Le sujet de l'énonciation se départit ainsi de toute assignation identitaire liée à sa généalogie biologique car son corps n'est « pas simplement la sédimentation des actes de discours qui l'ont constitué. Cette constitution peut échouer, lorsque l'interpellation rencontre une résistance au moment où elle veut imposer ses exigences ${ }^{22}$ ». Cette résistance, Idriss la fait sienne.

\section{La marge : un contre-discours}

Les romans dont il est question ici se positionnent dans la société en tant que contre-discours qui pensent les pratiques sexuelles comme des relations et non comme des formes. Ces textes se démarquent des définitions légitimes et dominantes de l'hétéronormativité. Ce n'est plus le destin d'une collectivité qui est mis en scène par ces auteurs, mais plutôt une expérience individuelle qui tente de se dire malgré la coercition qu'exerce le discours dominant sur le sujet de l'énonciation. L'un de ces auteurs, Ibrahim Bouzalim, touche à la cohésion religieuse, politique et sociale de la société marocaine puisqu'il dénonce dans ses deux volumes autobiographiques, Ramadu Souss (Cendres du Souss) et Moustanqa'at Souss (Les Mares du Souss), le directeur d'un Institut d'enseignement fondamental ${ }^{23}$ qui abuse sexuellement de ses élèves internes. Ce directeur, sur-nommé La Pieuvre (Al-Okhtobout), détient un pouvoir qui dépasse celui de l'État, représenté par le gouverneur de la région. Celui-ci invite Si Mohamed, fraîchement muté à la direction de 1'Institut fondamental de Tiznit après avoir exercé longtemps à Taroudant la même fonction et lui fait comprendre que Tiznit est une ville calme et qu'il serait bien d'éviter les mœurs auxquelles il était habitué à Taroudant ${ }^{24}$. En guise de réponse, le directeur interpelle le gouverneur dans les termes suivants :

— Tu es d'où, toi?

- De Berchid, Monsieur.

- Ah Berchid, la ville des fous et des aliénés. Tu dois être certainement croyant. Tu dois prier, jeûner, vénérer Allah et les Grands et ne perdre aucune occasion de faire l'aumône.

22. Judith Butler, Le Pouvoir des mots : politique du performatif [1997], trad. de Charlotte Nordmann, Paris, Éditions Amsterdam, 2004. J. Butler s'inspire ici des travaux de Louis Althusser dans son usage de la notion d'interpellation. Voir aussi Défaire le genre, Paris, Éditions Amsterdam, 2006.

23. C'est un établissement de l'enseignement secondaire qui se trouve dans le Sud marocain et qui dispense un enseignement d'obédience arabophone avec une dominante de la théologie musulmane. Le français y est enseigné en tant que matière secondaire.

24. Le gouverneur fait ici allusion aux pratiques homosexuelles actives du directeur. 
- Un peu, monsieur...

— Et tu forniques avec les prostituées?

- Il y a longtemps, avant de me marier!

— Et tu le fais encore. Il n'y a pas quelqu'un de censé comme toi qui pense m'intimider. Je suis Allah, le ciel, le paradis, l'enfer et la terre. Je suis la mort, la vie, la terreur, la négation et le recruteur des fonctionnaires ${ }^{25}$ !

À cette dénonciation des hommes censés représenter l'ordre, s'ajoute chez Karim Nasseri la destruction de l'image du père dans Noces et funérailles. Le père est sur-nommé Dictateur (dénomination qui le caractérisait déjà dans un précédent roman intitulé Chronique d'un enfant $d u$ hammam $^{26}$ ) par son propre fils, le narrateur Idriss, qui lui voue une haine incommensurable :

J'étais écœuré par l'attitude du Dictateur qui s'absentait une année entière
en nous laissant dans la misère totale puis revenait m'offrir des sucreries ${ }^{27}$.

La critique de la figure paternelle est accompagnée d'une critique politique. L'usage même du mot Dictateur inscrit le père dans un champ lexical du pouvoir politique. Il est, pourrait-on dire, le prisme par lequel passe le système de gouvernance qui régit toute la société du roman. L'assujettissement de l'enfant et l'investissement de son corps sont mis en texte à travers ce père castrateur. La sexualité d'Idriss est un moyen d'échapper à cette dictature polycéphale qui oppresse ses désirs non seulement sexuels, mais aussi culturels puisque son père voulait à plusieurs reprises l'empêcher de poursuivre sa scolarité :

Idriss peut bien se passer d'école. Il est né dans ce village entre les pattes des mules et des vaches, il mourra dans ce village entre les pattes des mules et des vaches ${ }^{28}$.

Contrairement au contexte américain qui reconnaît l'existence d'une communauté homosexuelle, le contexte marocain feint de l'ignorer tout simplement. En théorie, l'homosexualité est reléguée au rang de l'anomalie, voire condamnée juridiquement au nom de la morale religieuse, mais dans les faits, la société donne l'impression de la tolérer. Abdellah Taïa, Rachid O., Karim Nasseri, Ibrahim Bouzalim et Nedjma mettent en texte « la technologie du pouvoir sur le corps » (M. Foucault) en mettant en scène des pratiques sexuelles marquées par la marginalité. Cependant, cette tolérance non avouée s'inscrit dans la logique de l'exclusion et de la marginalisation.

25. Ibrahim Bouzalim, Moustanqa'at Souss (Les Marres du Souss), Dchira, Imprimerie Algamani, 1999, p. 17-18. Nous traduisons.

26. Karim Nasseri, Chronique d'un enfant du hammam, Paris, Denoël, 1998.

27. Karim Nasseri, Noces et funérailles, op. cit., p. 22.

28. Ibid., p. 113. 


\section{Corps écrit, corps censuré}

Les textes étudiés ici (mis à part quelques séquences de L'Amande, Ramadu Souss et Moustanqa'atu Souss) procèdent à un refoulement à travers l'euphémisme énonciatif qui marque les pratiques sexuelles mises en scène. Il est frappant de constater la quasi-absence de scènes textuelles décrivant des homosexuels nus : le corps homosexuel semble frappé d'une autocensure qui interdit la visibilité de sa nudité. Rachid O. évoque ses désirs et ceux de ses amants sans les décrire, Karim Nasseri (le plus permissif avec Nedjma et Ibrahim Bouzalim) parle des éjaculations du Fquih (maître de l'école coranique) sans faire voir au lecteur ce que celui-ci lui a appris lors de son initiation sexuelle (l'expression est du narrateur, p. 14) et la romancière Bahaa Trabelsi relate dans Une vie à trois ${ }^{29}$ l'histoire du couple Adam-Jamal mais ne procède à aucun moment à la représentation de leur vie sexuelle. Dans tous les cas évoqués ci-dessus, tout se passe comme si parler des anormaux était déjà excessivement osé : évoquer l'homosexualité, le lesbianisme, la pédérastie et les pratiques pornographiques, sans les inscrire dans un discours descriptif, semble être déjà subversif en soi. Nul besoin donc d'aller plus loin dans la représentation de l'acte sexuel proprement dit : les auteurs ne s'attardent pas sur la mise en scène des désirs charnels socialement pervers. La perspective descriptive est ainsi occultée au profit d'une perspective narrative qui informe sans trop représenter. La narration laisse au lecteur le loisir de se représenter ou non l'acte sexuel occulté par le narrateur. L'interdit semble venir ici de la relation psychique que le moi du narrateur (et par ricochet celui de l'auteur qui a organisé discursivement l'univers du récit avant de le mettre en circulation dans l'espace social) entretient avec sa propre sexualité.

C'est ainsi que, malgré certaines scènes osées, Rachid O. n'a pas surmonté sa censure psychique puisque ses textes narrativisent la relation homosexuelle de manière pudique. Même les scènes qu'il évoque sont très laconiques et se limitent, le plus souvent, à des attouchements ou à des expressions comme « nous avons fait l'amour ». Nouâmane, l'un des personnages de Ce qui reste, tout en prétendant que parler de sa vie et de sa sexualité est pour lui « une manière de faire de la pudeur une hypocrisie ${ }^{30} »$, produit un discours euphémique sur ses pratiques et ses choix sexuels. Sa sexualité et celle du narrateur ne sont pas suffisamment mises en discours par le langage et c'est là où la censure intervient puisque le sexe et ses enjeux de pouvoir sont sous l'emprise de la litote. Mais c'est en même temps cette évocation subtile du sexe qui tisse une connivence entre l'auteur et son lecteur. On pourrait soumettre ici une hypothèse : la limite de ces textes réside dans leurs stratégies énonciatives qui visent essentiellement à transgresser l'hétéronormativité dans un contexte culturel où la transgression, en générale, n'est pas considérée comme valeur constructive susceptible de participer au renouvellement de la société.

29. Bahaa Trabelsi, Une vie à trois, Casablanca, EDDIF, 2000.

30. Rachid O., Ce qui reste, Paris, Gallimard, coll. « L'Infini », 2003, p. 50. 
En effet, les narrateurs des romans cités plus haut n'évoquent que de manière allusive et dans un discours pudique et bref leurs actes sexuels. Ce refoulement est à mettre au compte de l'éducation sexuelle de ces auteurs en tant que sujets (au sens psychanalytique) marocains marqués par la censure du désir homosexuel. Ce refoulement apparaît chez Abdellah Taïa à travers son narrateur homodiégétique attiré par son camarade de classe Oussama :

$\mathrm{Au}$ cours des trois années scolaires qui suivirent, ma passion pour Oussama est restée la même, toujours intense, mais avec le temps j'ai appris à la cacher, à prendre de la distance, à souffrir seul, en silence ${ }^{31}$.

Ibrahim Bouzalim est cependant allé plus loin dans la transgression de la censure descriptive en associant de manière systématique la transgression de la représentation sexuelle et la transgression des tabous politiques. Ce procédé est configuré dans la narration à travers la mise en scène des pratiques sexuelles des hommes de pouvoir (Fquih, élus locaux, députés, riches...) et la dénonciation de l'usage que font ces mêmes hommes du pouvoir qui leur est octroyé (pouvoir symbolique ou réel).

Le potentiel subversif de ces récits littéraires ne réside pas nécessairement dans leur poéticité. Il est plutôt lié à leur fonction thématique qui, justement, leur accorde une originalité dans le champ littéraire marocain. Cette fonction déjoue la censure qui jette tout ce qui n'est pas hétéronormatif hors du dicible et donc, hors de l'espace social. En tant qu'acte de discours, les récits pris en charge par les narrateurs-personnages s'inscrivent dans « un rite d'institution » : ce sont des récits qui tentent d'arracher au sexe sa vérité. Ils sacrifient parfois l'élaboration stylistique au profit de la mise en scène d'un vécu frappé d'interdit. Chaque narrateur déclare, en contournant la censure, les éléments constitutifs de son identité sexuelle. Il semble vouloir dire à travers son récit : voici « la forme de mon existence », pour reprendre une expression utilisée par Louis Althusser dans son autobiographie ${ }^{32}$.

Le premier roman de Mohamed Leftah, passé par ailleurs inaperçu au Maroc, porte le titre énigmatique de Demoiselles de Numidie ${ }^{33}$. Une édition marocaine de ce récit, flamboyant et corrosif, vient de paraître chez Tarik Éditions. Le titre Demoiselles de Numidie renvoie à une formule par laquelle les Romains désignaient les grues; ces oiseaux carnivores et migrateurs dont les longues pattes leur permettent de traverser les fonds vaseux des marais. Mais, dans le langage populaire, le mot grue signifie prostituée. Par un jeu d'analogie ambiguë, Mohamed Leftah annonce, dès le titre, les figures principales et anti-héroïques de son roman : Nouar, Warda, Massc Allill, Krounfoul, Zoumourroud et bien d'autres filles de joie qu'il appelle si poétiquement « les filles-fleurs ». Ce qui intéresse l'auteur ce ne sont pas tant les marginalités

31. Abdellah Taïa, Mon Maroc, Paris, Séguier, 2000, p. 88.

32. Louis Althusser, L'Avenir dure longtemps, Paris, Stock/IMEC, 1992.

33. Mohamed Leftah, Demoiselles de Numidie, Paris, L'Aube, 1992 (réédité aux Éditions de la différence, coll. « Minos » en 2006). 
sociales que l'ordre poétique qu'on peut extraire de la « laideur » et des tristes beautés. Au-delà du sujet de ce roman, l'auteur met en scène, de manière réflexive, la relation palimpsestueuse entre les langues arabe et française. Le narrateur transforme les prénoms en objet de réflexion pour livrer au lecteur ses pensées « philologiques » sur les potentialités de sens que le corps des personnages entretient avec les noms qu'ils portent : « Nouar, pluriel de fleur, est le nom dont on désigne la syphilis, en cette vielle terre de Numidie, maladie qui longtemps habita le corps et hanta l'imagination des hommes » (p. 9). Le beau (la fleur) s'inscrit ainsi dans une esthétique du laid (la syphilis) pour estomper les frontières du goût. Les personnages principaux sont par ailleurs des prostituées définies, à chaque fois, par les prénoms qu'elles portent. Il n'y a pas de déterminisme dans ce lien établi entre elles et leurs prénoms, mais plutôt un écart : elles portent des prénoms socialement valorisés, mais assument une fonction socialement et moralement dégradante. Le beau donne ainsi naissance au laid pour rappeler des aspirations esthétiques déjà développées par l'auteur des Fleurs du mal que Leftah cite avec délectation. Tout en prenant le langage pour objet de réflexion, cet amoureux de la langue inscrit son œuvre dans la filiation littéraire qui a façonné son imaginaire et a fait de lui un écrivain sans épithète ni complément. Le lieu même où se passe l'action de son roman $A u$ Bonheur des limbes est éminemment ancré dans l'imaginaire littéraire moderne : Don Quichotte. Là encore l'ambiguïté, condition de toute création littéraire, est maintenue. S'agit-il du livre de Cervantès? Il s'agit du nom d'un bar situé en plein centre de Casablanca. Pour un lecteur (et c'est le plus important) qui se laisse saisir par le plaisir de la langue, la poétique du titre transforme l'univers de la « Fosse » en un lieu investi de poésie. Ainsi les corps qui s'y trouvent deviennent eux-mêmes objet de poésie.

« Les petits fait vrais du passé » que Leftah a vécus, vus ou entendus constituent la matière de ses livres. C'est en partant de l'un de ces faits qu'il livre dans Une Chute infinie (2009), récit sous-titré Petite chronique en référence à l'écrivain sicilien Leonardo Sciascia, l'histoire de Khalid (un adolescent « au corps sculptural d'athlète ») qui a mis fin à sa vie devant le regard médusé de son professeur M. Ciccion et de ses camarades de classe. C'était à Settat, ville natale de l'auteur. Khalid ouvre la porte de la classe, « prend son élan en deux pas dansants et saute par-dessus le parapet » (p. 31). Cette petite chronique poétique est surtout un hymne au corps avec toutes ses ambiguïtés. Au mois de février 2010, deux autres ouvrages posthumes ont été publiés par le même éditeur : Hawa (roman) et Récits du monde flottant (recueil de nouvelles). Hawa, Amour, et Zapata, de son vrai nom Mahboub, sont des jumeaux incestueux et « de monstrueuse beauté » (p. 43). Leur mère n'est autre que la fameuse Warda qu'on retrouve dans Demoiselles de Numidie et Au Bonheur des limbes. Zapata, proxénète professionnel et amant du Norvégien Ingvar, est également l'un des anti-héros de Demoiselles de Numidie. Sa sœur Hawa, cette femme 
encore si jeune ravie à elle-même, est qualifiée de mejdouba ${ }^{34}$ (p. 122). Son statut marginal devient ainsi similaire à celui des mystiques qui sont, du moins pour les rigoristes orthodoxes, à la marge de l'islam. Les jumeaux sont nés dans le fameux quartier Boussbir, bordel casablancais à ciel ouvert, dont le nom est :

\begin{abstract}
la déformation d'un nom propre, celui d'un certain M. Prosper, un Français à l'origine de l'érection de ce quartier qui approvisionna longtemps en chair fraîche et constamment renouvelée des clients qui pouvaient être des indigènes, mais en premier et surtout les soldats de la France, pays qui colonisait alors le Maroc, ensuite, lors du débarquement américain à Casablanca pendant la Seconde Guerre mondiale, ces soldats à la carrure de géants et au sourire d'enfants qui, en groupe, bruyants et sans la moindre gêne, baisaient comme des forcenés la putain indigène.(p. 14)
\end{abstract}

Le narrateur, qui se présente au lecteur comme un scribe, tisse l'intrigue de son récit avant tout par sa culture encyclopédique : les films La Révolte de Spartacus et Viva Zapata, les livres mystiques, Othello de Shakespeare, Le Livre de la pauvreté et de la mort de Rainer Maria Rilke, le mouvement artistique et poétique Cobra... Il s'agit donc de dire le réel le plus ténébreux de l'homme par la médiation du langage poétique. Pour le scribe, il ne s'agit pas de réhabiliter le quartier Boussbir, mais plutôt de faire briller « à nouveau et de mille éclats, le feu du désir et du poème, aussi sacré que celui à l'entretien duquel étaient préposées les antiques et hiératiques vestales » (p. 110).

Que ce soit le roman au féminin ou l'écriture de l'homoérotisme, il s'agit toujours d'une mise en scène de la technologie du pouvoir sur les corps culturellement et socialement indexés au moindre écart par rapport à la loi phallocratique. L'impensé de cette « marginalité » à laquelle s'expose toute déviation « corporelle » inscrit une tension narrative dans les récits littéraires marocains et leur confère, par là même, une fonction de résistance aux discours ambiants qui naturalisent ce qui relève de la culture et de l'histoire. Inscrire le corps « enfermé » et « marginal » dans les textes littéraires, c'est lui donner la possibilité de dire les ambiguités de toute une société fondée sur la métaphysique des séparations. Les romans analysés ci-dessus déconstruisent les oppositions entre féminin et masculin, dominant et subalterne, homme et femme. De ce point de vue, ils assument une fonction non seulement esthétique, mais aussi épistémologique.

Khalid Zekri Équipe d'Études Culturelles et Postcoloniales - Université de Meknès

34. Majdouba en arabe littéraire ou mejdouba en arabe parlé/dialectal, désigne une femme ayant des pouvoirs de sorcellerie, de voyance et de guérison. Elle peut en particulier pratiquer des envoûtements et des contre-envoûtements. Le terme peut désigner aussi une femme possédée,ou ensorcelée. 